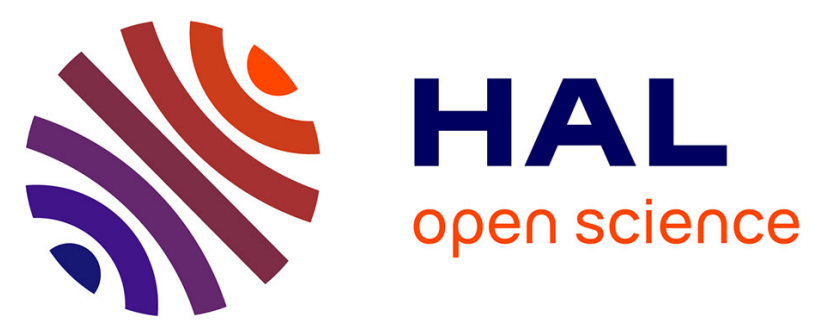

\title{
Land Seismic Multi-Parameter FWI in Elastic VTI Media by Simultaneously Interpreting Body Waves and Surface Waves
}

Weiguang He, Romain Brossier, Ludovic Métivier, René-Edouard Plessix

\section{To cite this version:}

Weiguang He, Romain Brossier, Ludovic Métivier, René-Edouard Plessix. Land Seismic MultiParameter FWI in Elastic VTI Media by Simultaneously Interpreting Body Waves and Surface Waves. 81st EAGE Conference and Exhibition 2019, Jun 2019, London, United Kingdom. 10.3997/22144609.201900876 . hal-02325637

\section{HAL Id: hal-02325637 \\ https://hal.science/hal-02325637}

Submitted on 2 Dec 2020

HAL is a multi-disciplinary open access archive for the deposit and dissemination of scientific research documents, whether they are published or not. The documents may come from teaching and research institutions in France or abroad, or from public or private research centers.
L'archive ouverte pluridisciplinaire HAL, est destinée au dépôt et à la diffusion de documents scientifiques de niveau recherche, publiés ou non, émanant des établissements d'enseignement et de recherche français ou étrangers, des laboratoires publics ou privés. 


\title{
Land seismic multi-parameter full waveform inversion in elastic VTI media by simultaneously interpreting body waves and surface waves with optimal transport based objective function
}

\author{
Weiguang $\mathrm{He}^{1 *}$, Romain Brossier ${ }^{1}$, Ludovic Métivier ${ }^{1,2}$, and René-Édouard Plessix ${ }^{3}$ \\ ${ }^{1}$ Univ. Grenoble Alpes, ISTerre, 38000 Grenoble, France \\ ${ }^{2}$ Univ. Grenoble Alpes, CNRS, ISTerre, 38000 Grenoble, France \\ ${ }^{3}$ Shell Global Solutions International, Grasweg 31, 1031HW Amsterdam, The Netherlands \\ Weiguang.He@univ-grenoble-alpes.fr
}

January 13, 2019

\begin{abstract}
Land seismic multi-parameter full waveform inversion in anisotropic media is challenging because of high medium contrasts and because of surface waves. With a data-residual least-squares misfit function, the surface wave energy usually masks the body waves and the gradient of the misfit function exhibits high values in the very shallow depths preventing from recovering the deeper part of the earth model parameters. An optimal transport objective function, coupled with a Gaussian time-windowing strategy, allows to overcome this issue by more focusing on phase shifts and by balancing the contributions of the different events in the adjoint-source and the gradients. First, we show how OT function balances events. We then discuss a vertical transverse isotropic (VTI) example starting from a quasi 1D isotropic initial model. Despite some cycle-skipping issues in the initial model, the inversion based on the windowed optimal transport approach converges. Both the near-surface complexities and the variations at depth are satisfactorily recovered.
\end{abstract}




\section{Introduction}

Land seismic records contain both deep propagating body waves and shallow-penetrating but strongly energetic surface waves. When the latter are included in full waveform inversion (FWI), conventional misfit function (and inversion) is driven to update mainly the very shallow part (mostly for S-wave velocity), while the deeper subsurface structures of interest are weakly updated. In order to highlight the contribution from the deep-penetrating body waves, surface waves should be removed from the data by processing or specific treatment (Plessix and Pérez Solano, 2015).

Recently, an optimal-transport-based (OT) objective function has been proposed in the frame of acoustic FWI. The original motivation for OT in FWI is the convexity of the objective function with respect to shifted patterns, seen as a proxy of convexity with respect to velocity changes, in order to mitigate the cycle-skipping issue (Métivier et al., 2018; Yang et al., 2018). In this study, we exploit the interesting property of the Kantorovich-Rubinstein OT (Métivier et al., 2016; Poncet et al., 2018) to balance the amplitudes of various arrivals. The amplitude distribution of the OT adjoint source is more uniform than that of the least-squares function, suggesting that the high amplitudes of surface waves will have less impact into the misfit function and the gradient build-up. Besides, the OT adjoint source is less oscillating than the synthetics or observed data. For this reason, we investigate the behaviour of OT for this problem of imaging complex near-surface anisotropic targets.

In this study, the combined interpretation of surface waves and body waves from a synthetic land dataset is investigated, using a multi-parameter anisotropic FWI scheme based on an optimal transport (OT) misfit function. We show that this approach combined with a Gaussian time window to damp far-offset surface waves allows the reconstruction of meaningful information on $\mathrm{P}$ and S-wave velocities as well as on the anisotropy parameters.

\section{Method}

\section{Theory}

Following Métivier et al. (2016), the OT objective function with the Kantorovich-Rubinstein approach is based on the dual of the 1-Wasserstein distance. For a shot indexed by $s$, the corresponding misfit is

$$
h_{s}\left(d^{s y n}, d^{o b s}\right)=\max _{\varphi_{s} \in \operatorname{BLip}_{1}} \sum_{r} \int_{0}^{T} \varphi_{s}\left(x_{r}, t\right) W_{s r}(t)\left[d_{s r}^{s y n}(t)-d_{s r}^{o b s}(t)\right] \mathrm{d} t \Delta x_{r},
$$

where $\Delta x_{r}$ is the inter-receiver distance, $W_{s r}(t)$ is a weighting function introduced to represent the timewindowing technique, and the signed residuals $\delta d_{s r}=d_{s r}^{s y n}-d_{s r}^{o b s}$ are the differences between synthetic and observed data traces. BLip 1 is the space of Bounded 1-Lipschitz functions defined in the $\left(x_{r}, t\right)$ shot-gather space by

$$
\left|\varphi_{s}\right|<\lambda, \quad\left|\nabla_{x} \varphi_{s}\right|<1, \quad\left|\nabla_{t} \varphi_{s}\right|<1,
$$

where $\lambda$ is a pre-defined constant to ensure that $\varphi_{s}$ does not go to infinity, while $\nabla_{x}$ is the first order derivative operator in $x$ direction, and the same for $\nabla_{t}$ in $t$ direction. The first inequality ensures the amplitudes of $\varphi_{s}$ are bounded; the last two inequalities prevent abrupt variations of the solution $\varphi_{s}$. It is these inequalities that ensure the solution $\varphi_{s}$ is uniform. The maximization problem (Equation 1) can be solved efficiently through proximal splitting techniques (Métivier et al., 2016). Once the optimal $\bar{\varphi}_{s}\left(x_{r}, t\right)$ is determined, the corresponding adjoint source is simply

$$
\mathscr{D} h_{s}=\bar{\varphi}_{s}\left(x_{r}, t\right) W_{s r}(t) \text {. }
$$

\section{Role of the bound constraints}

The shape of the OT adjoint source depends on whether the bound constraint $\lambda$ is bigger or smaller than the smoothing constraint 1 in Equation 2. We use a $3 \mathrm{~Hz}$ Ricker wavelet to illustrate this. The calculated trace is normalized to 1 , while the observed trace is intended to be smaller with a maximum amplitude of 0.2 (Figure 1 (a)). When the bound constraint $\lambda$ is smaller than 1 , the OT adjoint source mimics a least-squares adjoint source (Figure 1 (b)). For values of $\lambda$ larger than 1, the OT adjoint sources are similar to each other and display balanced amplitudes of the different events (Figure 1 (b)). In realistic situations, we could have missing events and/or misaligned events. Figure 1 (c) and (d) show that in 


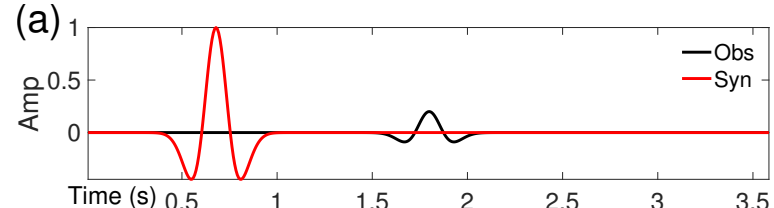

(b)

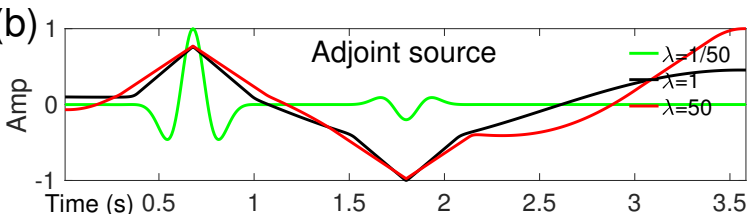

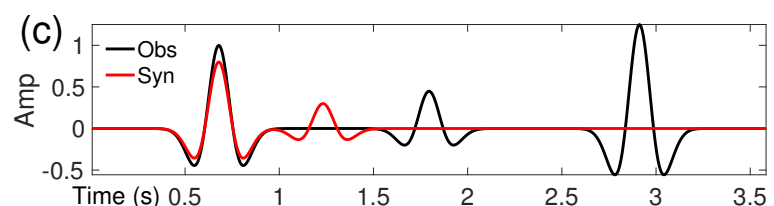

(d)

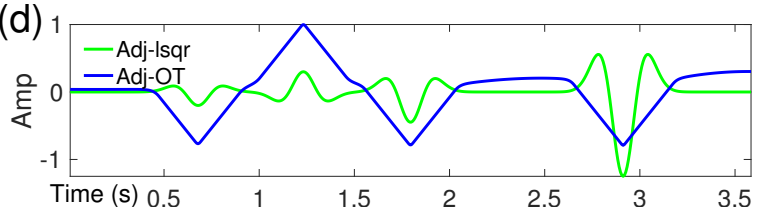

Figure $1(a)$ and (b) illustrate the effects of bound constraints $\lambda$ on the OT adjoint source. (c) and (d) show the case of multiple events.

such a more complex situation, the OT function still balances the amplitudes of the events in the adjoint source, where small signals are enhanced and the large events are decreased.

\section{Inversion of a challenging VTI example}

A realistic representation of the Earth is to consider elasticity and anisotropy. To this purpose, a VTI subsurface structure has been created from the SEAM II Foothill model. The original varying topography is flattened but the near-surface complexity is kept. Five parameters $v_{n}, v_{s}, \rho, \eta$ and $\delta$ are shown in Figure 2. The initial model is quasi-1D isotropic (Figure 2). We show the observed and synthetic seismograms in Figure 3. The observed data contain numerous scattered events. The effects of low-velocity zones on the early body waves can be identified around $X=6 \mathrm{~km}$ and $X=9 \mathrm{~km}$ in Figure 3 (a). The surface waves are strongly affected by the near-surface complexity, and they are strongly scattered around $X=10 \mathrm{~km}$. In comparison, the initial synthetics display much simpler waveforms.

Four parameters $\left(v_{n}, \eta, \delta, v_{s}\right)$ are simultaneously updated while density is calculated from $v_{n}$ at each iteration. First, we present the inversion obtained by applying the least-squares function and Gaussian time windows with standard deviation $\sigma=1.6 \mathrm{~s}$. Even though the model is mainly updated in the shallow part, the structures could be recognized.

We apply the OT function using the same Gaussian window with $\sigma=1.6 \mathrm{~s}$, and get a subsurface structure shown in Figure 5. The velocity parameters $v_{n}$ and $v_{s}$ are well recovered. We can see the low-velocity zone, near-surface complexity, interior low-velocity channels. The final synthetics capture most of the features of the true seismogram. Figure 6 shows the trace comparison at $X=8 \mathrm{~km}$ and $X=11 \mathrm{~km}$. Even when there is significant cycle-skipping for both body waves and surface waves in the initial model, we are able to fit them at the end of the inversion.

\section{Discussion and conclusions}

The OT strategy is very attractive for elastic FWI mainly because this misfit function is able to balance energy among various events. In the gradient build-up, the contribution from surface waves and body waves is balanced. Therefore, a conventional two-stage strategy, where body waves are fitted first before including surface waves, is not required with the OT function. With a Gaussian window, the OT function performs quite robustly. It is nevertheless important to consider a smooth window for three reasons. First, an OT algorithm compares patterns. With smooth windows, the shape of events are preserved. Second, damping the long-offset surface waves reduces the risk of cycle-skipping. Third, most importantly, keeping the near-offset events (shear waves and surface waves) helps to keep a balanced contribution between body waves and surface waves. For the anisotropic SEAM II Foothill model, the velocity parameters are well recovered. The anisotropy parameters are not recovered with same quality for the velocity parameters. The anisotropy parameters are over-estimated, but the structures are revealed. We also tested the OT function without applying a Gaussian time window. The surface waves are so dominant that only $v_{s}$ is mainly updated, and its resolution is much lower compared to $v_{s}$ in Figure 
(a-1)

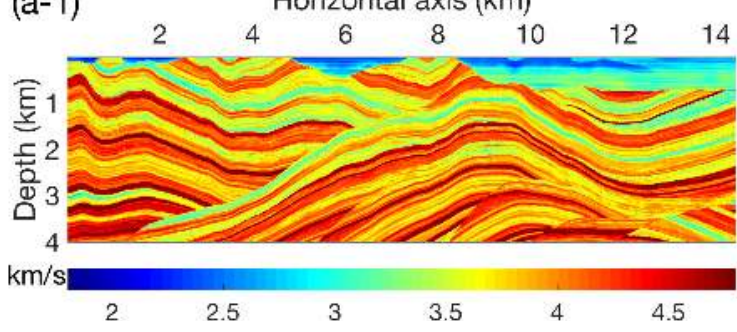

(b)

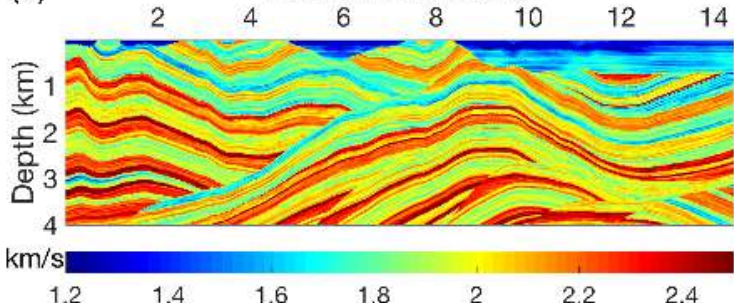

(d)

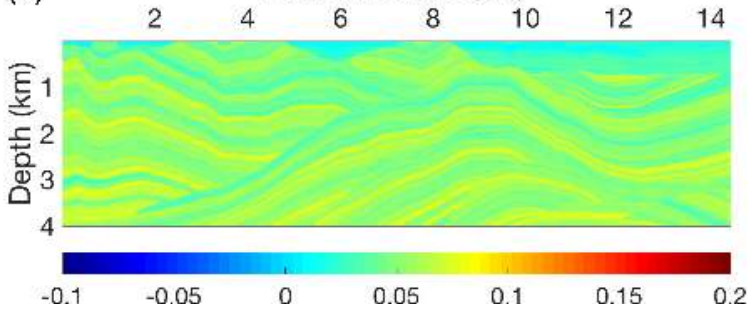

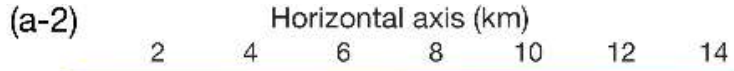
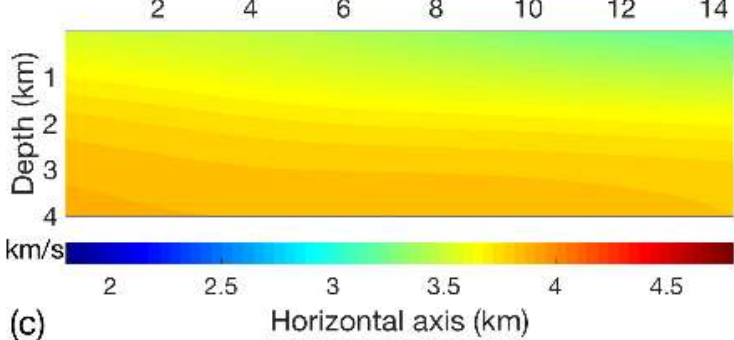

(c)

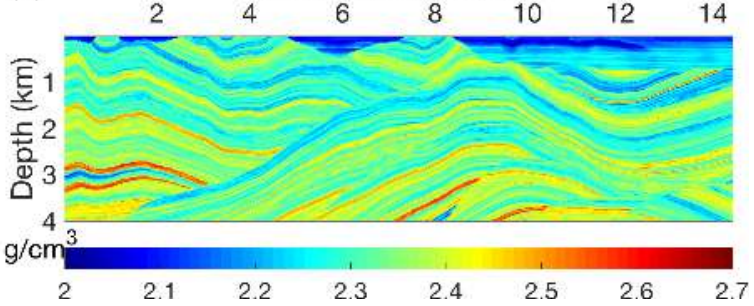

(e)

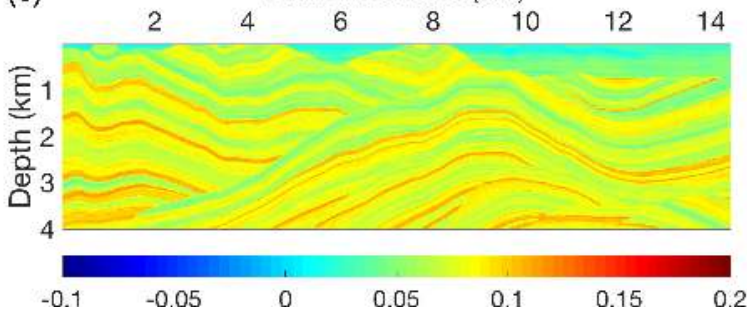

Figure 2 True 2D VTI SEAM II Foothill model and initial smooth isotropic model. (a-1) True $v_{n},(a-2)$ Initial $v_{n},(b)$ True $v_{s},(c)$ True $\rho,(d)$ True $\eta$, (e) True $\delta$. Initial $\rho=\rho\left(v_{n}\right)$ is calculated from initial $v_{n}$, initial $\eta$ and $\delta$ are 0 .

(a)

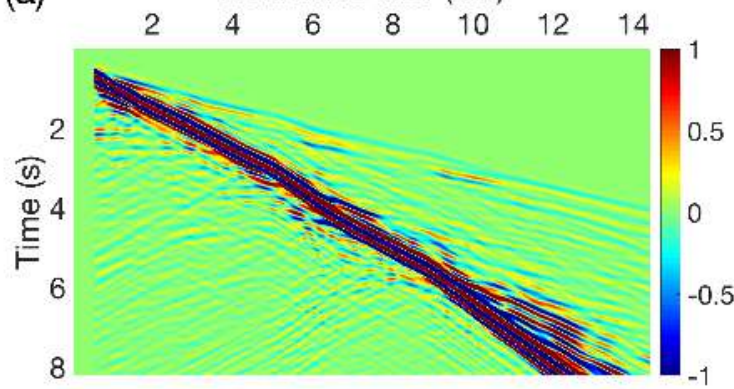

(b) Horizontal axis (km)

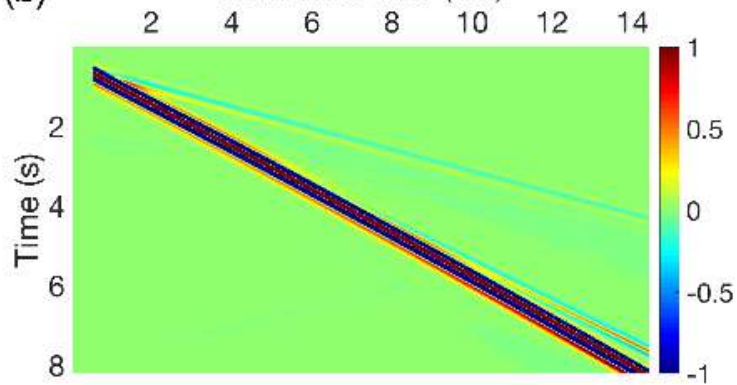

Figure 3 An observed and initial shot gather with the anisotropic SEAM II Foothill model shown in Figure 2. (a) Observed data and (b) Initial synthetics. Cycle-skipping ocurrs for both body waves and surface waves.
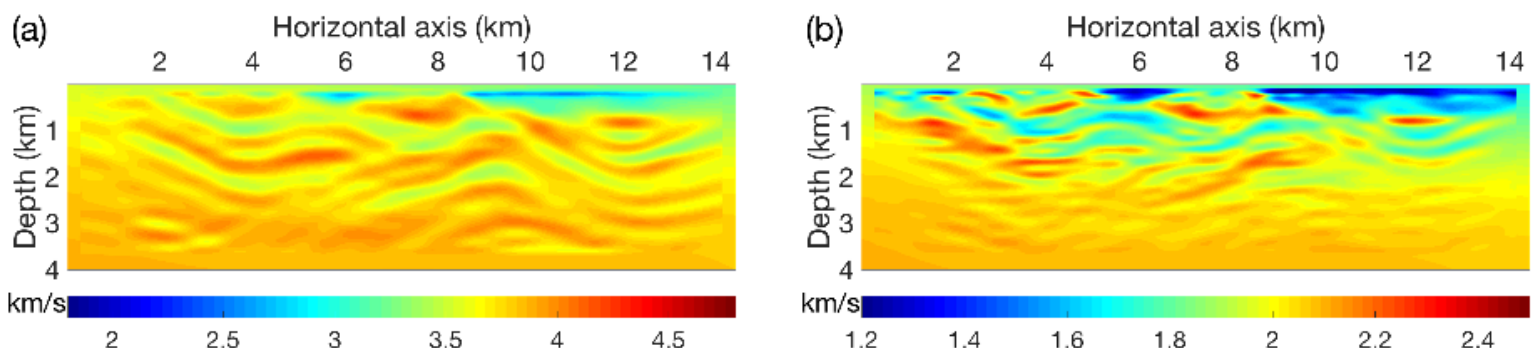

Figure 4 Inversion results of the anisotropic SEAM II Foothill example with a least-squares function and a Gaussian window with $\sigma=1.6 s$. (a) $v_{n}$, (b) $v_{s}$. 

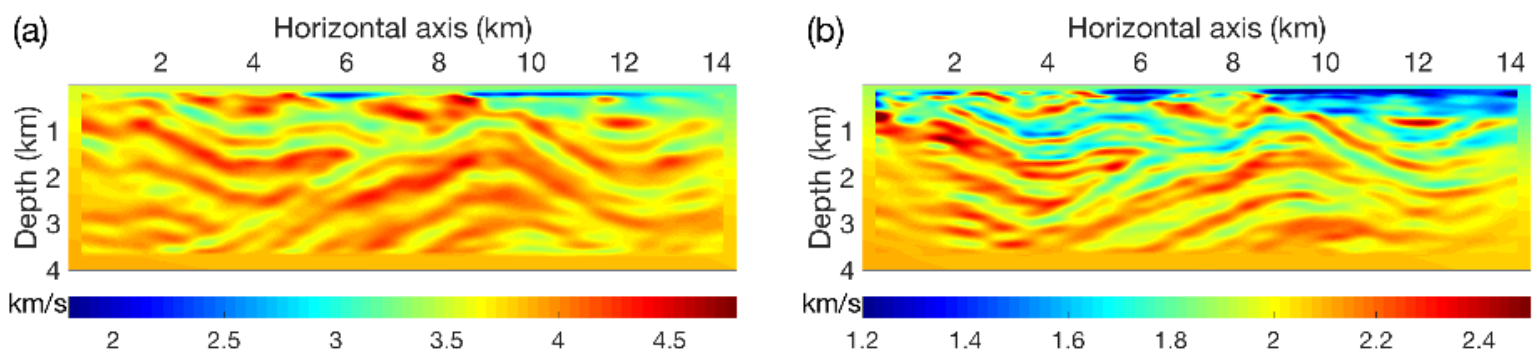

Figure 5 Inversion results of the anisotropic SEAM II Foothill example with the OT function and a Gaussian window with $\sigma=1.6 s$. (a) $v_{n}$, (b) $v_{s}$.
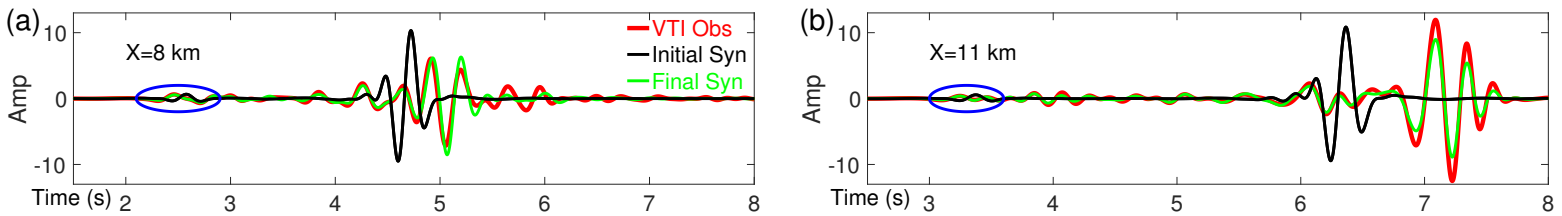

Figure 6 Data trace comparisons (a) at X=8 km and (b) at X=11 km. Initially cycle-skipped surface waves and body waves are fit after inversion.

5 (b).

The design of the smoothing window is similar to the strategy of Brossier et al. (2009). Although in principle we could also design a second level loop over the Gaussian windows besides the frequency continuation strategy, we choose not to do so in this study. We aim to demonstrate that a multi-parameter inversion is possible when the surface waves and body waves are suitably balanced. To illustrate the relevance of our inversion strategy, we carried out some inversions with the Middle East model (He et al., 2018), which is a different geologic complexity than the elastic SEAM II Foothill model. While SEAM II Foothill model produces significant reflections and diffractions without deep-penetrating diving waves, the Middle East model produces abundant diving waves and near surface reflections. With our OT based strategy, the model is also well retrieved.

\section{Acknowledgements}

This study was partially funded by the SEISCOPE consortium (http://seiscope2.osug.fr), sponsored by AKERBP, CGG, CHEVRON, EQUINOR, EXXON-MOBIL, JGI, PETROBRAS, SCHLUMBERGER, SHELL, SINOPEC and TOTAL. This study was granted access to the HPC resources of CIMENT infrastructure (https://ciment.ujfgrenoble.fr) and CINES/IDRIS/TGCC under the allocation 046091 made by GENCI.

\section{References}

Brossier, R., Operto, S. and Virieux, J. [2009] Seismic imaging of complex onshore structures by 2D elastic frequency-domain full-waveform inversion. Geophysics, 74(6), WCC105-WCC118.

He, W., Plessix, R.É. and Singh, S. [2018] Parameterization study of the land multi-parameter VTI elastic waveform inversion. Geophysical Journal International, 213(3), 1662-1674.

Métivier, L., Allain, A., Brossier, R., Mérigot, Q., Oudet, E. and Virieux, J. [2018] Optimal transport for mitigating cycle skipping in full waveform inversion: a graph space transform approach. Geophysics, 83(5), R515-R540.

Métivier, L., Brossier, R., Mérigot, Q., Oudet, E. and Virieux, J. [2016] Measuring the misfit between seismograms using an optimal transport distance: Application to full waveform inversion. Geophysical Journal International, 205, 345-377.

Plessix, R.E. and Pérez Solano, C. [2015] Modified surface boundary conditions for elastic waveform inversion of low-frequency wide-angle active land seismic data. Geophysical Journal International, 201, 1324-1334.

Poncet, R., Messud, J., Bader, M., Lambaré, G., Viguier, G. and Hidalgo, C. [2018] FWI with Optimal Transport: a 3D Implementation and an Application on a Field Dataset. In: Expanded Abstracts, $80^{\text {th }}$ Annual EAGE Meeting (Copenhagen).

Yang, Y., Engquist, B., Sun, J. and Hamfeldt, B.F. [2018] Application of optimal transport and the quadratic Wasserstein metric to full-waveform inversion. GEOPHYSICS, 83(1), R43-R62. 